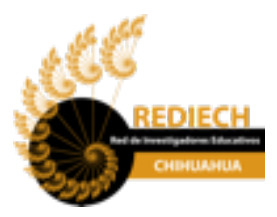

Red de Investigadores Educativos Chihuahua A.C. Chihuahua, México www.rediech.org

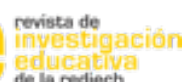

de la rediech

ISSN: 2007-4336

ISSN-e: 2448-8550

http://www.rediech.org/ojs/2017/index.php/ie rie rediech/index

Carlos Ramírez Silván

Silvia Patricia Aquino Zúñiga

2019

\title{
LOS ORGANISMOS INTERNACIONALES Y LAS POLÍTICAS EDUCATIVAS DE PROFESIONALIZACIÓN DOCENTE DE LA EDUCACIÓN NORMAL EN MÉXICO
}

IE Revista de Investigación Educativa de la REDIECH, 10(19), pp. 71-89.

DOI: http://dx.doi.org/10.33010/ie_rie_rediech.v10i19.515

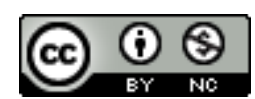

Esta obra está bajo licencia internacional

Creative Commons Reconocimiento-NoComercial 4.0.

CC BY-NC 4.0 


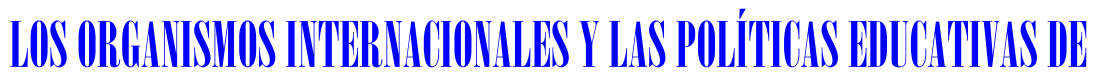

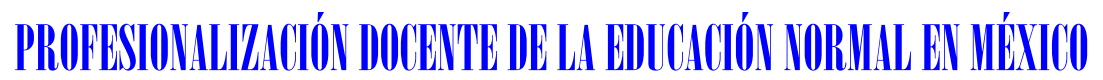

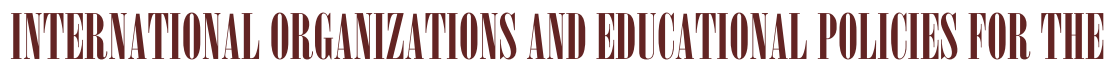

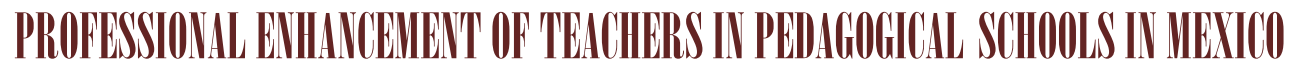

\author{
RAMÍREZ SILVÁN Carlos \\ AQUINO ZÚÑIGA Silvia Patricia
}

Recepción: diciembre 12 de 2018 | Aprobado para publicación: junio 23 de 2019

DOI: http://dx.doi.org/10.33010/ie rie rediech.v10i19.515

$\sim$

\section{Resumen}

Este artículo presenta los resultados de una revisión de la literatura sobre el papel que tienen los organismos internacionales en el establecimiento de las políticas de profesionalización docente de las escuelas normales en México. Se utilizaron las bases de datos Google Scholar, Dialnet, Redalyc, SciELO y Latindex de 1984 a 2018. La selección comprendió ensayos o estudios empíricos principalmente cualitativos, aunque no se descartaron los cuantitativos. Las políticas de profesionalización docente se han diseñado desde el enfoque del capital humano, la nueva gestión pública (NGP) y el gerencialismo. El propósito fue identificar las políticas educativas que se han impulsado en México para profesionalizar a los docentes de las escuelas normales y comparar las acciones que se

Carlos Ramírez Silván. Profesor-investigador de la Escuela Normal de Educación Preescolar Profra. Rosario María Gutiérrez Eskildsen, de Villahermosa, Tabasco, México. Es maestro en Ciencias de la Educación con Especialidad en Docencia e Investigación de la Educación Superior por la Universidad del Valle de México Campus Villahermosa. Tiene los reconocimientos al perfil Prodep y es miembro del Padrón Estatal de Investigación del Consejo de Ciencia y Tecnología del Estado de Tabasco. Ha presentado ponencias en congresos nacionales de investigación sobre educación normal y en los eventos del Consejo Mexicano de Investigación Educativa. Correo electrónico: carlos. ramirezsilvan@gmail.com. ID: http://orcid.org/0000-0002-9267-9406.

Silvia Patricia Aquino Zúñiga. Profesora-investigadora de la División Académica de Educación y Artes de la Universidad Juárez Autónoma de Tabasco, México. Es doctora en Ciencias de la Educación por la Universidad de La Habana, Cuba. Tiene los reconocimientos al perfil Prodep y del Sistema Nacional de Investigadores. Entre sus publicaciones recientes se encuentran la que derivó en la coordinación del libro Verano de la investigación científica. Cantera del talento científico en la Universidad Juárez Autónoma de Tabasco (2018). Ha sido responsable y colaboradora de proyectos con financiamiento externo como Fomix, Conacyt, INEE-Conacyt, Prodep. Líder del Cuerpo Académico Consolidado Innovación e Investigación Educativa. Correo electrónico: saquinozuniga@gmail.com. ID: http://orcid.org/0000-0002-7223-8582. 
han llevado a cabo con los principios de la NGP. En México se crearon cinco programas de profesionalización docente. El más prominente es el Programa para el Desarrollo Profesional Docente. La exploración reveló que estas políticas se han diseñado, desarrollado y evaluado desde los principios de la NGP; en ellos se establece que el Estado debe dejar paso a las fuerzas del enfoque gerencial para que controlen la dinámica de los procesos de la administración pública.

\title{
Palabras clave: POLÍTICA EDUCATIVA, POLÍTICAS PÚBLICAS, EDUCACIÓN SUPERIOR, ESCUELAS NORMALES, PROFESIONALIZACIÓN.
}

\begin{abstract}
This article presents the results of a review of the literature on the role of international organizations in the establishment of the teacher professionalization policies of the Pedagogical Schools in Mexico. The databases Google Scholar, Dialnet, Redalyc, SciELO, and Latindex from 1984 to 2018 were used. The selection included mainly qualitative empirical trials or studies, although quantitative ones were not ruled out. Teacher professionalization policies have been designed from the perspective of human capital, the New Public Management (NPM) and managerialism. The purpose was to identify the educational policies that have been promoted in Mexico to professionalize teachers in Pedagogical Schools and compare the actions that have been carried out with the principles of the NPM. In Mexico, five teacher professionalization programs were created. The most prominent is the Teacher Professional Development Program. The exploration revealed that these policies have been designed, developed and evaluated from the principles of the NPM, in them, it is established that the State must give way to managerial approach so that they control the dynamics of the processes of public administration.
\end{abstract}

Keywords: EDUCATIONAL POLICY, PUBLIC POLICIES, HIGHER EDUCATION, TEACHERS TRAINING COLLEGE, PROFESSIONALIZATION.

\section{ITTRounc(ción}

Este ensayo es el resultado de una revisión de la literatura publicada sobre el papel que tienen los organismos internacionales -como el Banco Mundial (BM), el Banco Interamericano de Desarrollo (BID), la Organización para la Cooperación y el Desarrollo Económicos (OCDE) y la Organización de las Naciones Unidas para la Educación, la Ciencia y la Cultura (UNESCO por sus siglas en inglés)- en el impulso de reformas estructurales en la administración pública de México a través de políticas educativas para la profesionalización docente de las escuelas normales.

El diseño, ejecución y evaluación de políticas públicas debe darse en un marco 72 de inclusión para toda la población, sin preferencias ideológicas ni partidistas, sin 
tomar en cuenta intereses particulares, con un intenso deseo de servicio, transparencia y compromiso (Vargas, 2007). Las políticas públicas se establecen mediante un proceso que inicia con la detección de algún problema o situación que es necesario atender; continúa con la ejecución y finaliza con la evaluación de los resultados que se han obtenido como parte del establecimiento de esas políticas 0 acciones que se han puesto en marcha para eliminar, disminuir o atender de manera parcial o total el problema. La importancia del análisis de las políticas públicas educativas radica en obtener datos que informen del modo en que se pueden mejorar las condiciones de vida de una población. Este tipo de información puede ser técnica para llevar a cabo la toma de decisiones o contribuir con mayor profundidad en la comprensión de las relaciones políticas de los grupos humanos (Ramírez y Jiménez, 2008).

Las políticas públicas, de acuerdo con Roth Deubel (2003, p. 117), son:

Un conjunto conformado por uno o varios objetivos colectivos considerados necesarios o deseables, de medios y acciones que son tratados, por lo menos parcialmente, por una institución u organización gubernamental con la finalidad de orientar el comportamiento de actores individuales o colectivos para modificar una situación percibida como insatisfactoria o problemática.

En el plano internacional, el establecimiento, desarrollo y ejecución de las políticas públicas en la mayoría de los países de América Latina se han hecho con base en acuerdos con organismos internacionales como el BM y el BID como financiadores. Las políticas emitidas por estas instituciones tienen como referente el enfoque del capital humano (Moreno, 2017) originado en el conductismo, pragmatismo (Canto, 2011) y en los postulados de la NGP (Pérez, 2013) con su modelo empresarial, en el que los ciudadanos se asemejan a clientes, por los que las instituciones de educación superior compiten entre sí (Chica, 2011; Parcerisa, 2016). Las políticas de gestión públicas, en este paradigma, se orientan de acuerdo con Barzelay (2013, p. 120) en la "planeación de gastos y gestión financiera, función pública y relaciones laborales, recaudación, organización y métodos, y auditoría y evaluación".

Del mismo modo, organismos como la OCDE y la UNESCO sirven como asesores. La OCDE fundamenta sus recomendaciones en la investigación, evaluación, formación de docentes y estudiantes en el enfoque por competencias (SEP, 2012 y 2018). Por su parte, la UNESCO aboga por la promoción de la paz y porque todos los niños accedan a la educación; sin embargo, el enfoque está dirigido en adaptar la administración pública de los países asesorados, como los sistemas educativos, a la dinámica de la globalización, como lo manifiesta el informe Delors (UNESCO, 1996), el cual constituye uno de los escritos más influyentes sobre educación a nivel mundial. Este prioriza el desarrollo de competencias que garantice el desarrollo de la ciencia y la tecnología en los países subdesarrollados, pero elude la formación del pensamiento crítico, analítico y reflexivo de los estudiantes, factores que necesariamente fomentan la identidad local como un proceso que propicia el desarrollo humano y de la colectividad; no obstante, lo que se privilegia es el desarrollo económico (Alcántara, 2008 y Moreno, 2017). 
Por lo tanto, a nivel internacional las políticas en educación son objeto de estudio y reflexión y se toma como punto de partida la pluralidad de sus resultados; entre ellos destacan la baja calidad y la persistencia de la inequidad. Los efectos suelen ser circunstanciales e incrementales por las condiciones imperantes en cada país, por lo que el conocimiento de lo que se experimenta en otras regiones permite obtener certidumbre para el establecimiento dinamizador de la participación de los actores que intervienen en este proceso, tales como la investigación, los que toman las decisiones y otros involucrados, desde el momento de definir el problema de política pública, su ejecución y evaluación. (Gajardo, 1999; Levin, 2010 y Hannaway 2009, citados en Del Castillo-Alemán, 2012).

Los estudios que se han hecho en relación con el análisis de las políticas públicas, que se han establecido en los países de Latinoamérica, apuntan a que el paradigma empresarial de la NGP es el que predomina en este campo. Así lo señala un estudio dirigido por Del Castillo-Alemán (2012), quien hizo una investigación de las reformas sobre el sector educativo que se han llevado a cabo en México en los últimos 20 años; este se hizo desde el enfoque de análisis denominado "investigación de políticas" en el que se analiza el tema de la calidad educativa a través de una estrategia desarrollada por el gobierno para modernizar la gestión del sistema desde el paradigma de la NGP. Lo mismo ocurre en la investigación cuantitativa efectuada por Simbaqueba (2016), en el que se hizo un análisis de las reformas para la modernización administrativa que a nivel constitucional se hicieron en las leyes y reglamentos de la administración pública en Colombia a fin de alinearlas con los postulados de la NGP, tales como la planeación, la gestión financiera, la gestión administrativa, el control y la evaluación. Un estudio más fue el de Parcerisa (2016), con un enfoque cualitativo de análisis de caso en el que se compararon dos escuelas en Cataluña, España, sobre la recontextualización de la autonomía escolar, aspecto que se desarrolla junto con la rendición de cuentas y que se privilegian en la NGP.

El objetivo general del presente ensayo es identificar las políticas educativas que se han impulsado en México para profesionalizar a los docentes de las escuelas normales y comparar las acciones que se han llevado a cabo con lo que se establece desde el paradigma de la NGP recomendado por los organismos internacionales que financian y asesoran las reformas estructurales que se han establecido en el Sistema Educativo Mexicano. Se hará una revisión de la literatura sobre las políticas educativas que se han impulsado en México de 1984 a 2018 para profesionalizar a los formadores de docentes de las escuelas normales y la forma en que estos organismos han intervenido en el establecimiento, desarrollo, análisis y evaluación de esas políticas.

\section{Renisín Trónile}

\section{Enfoumes teóricos: el enfoutue del capital humbano, el conductismo y el priagmatismo}

Las políticas educativas que se han establecido en la mayoría de los países de América Latina han tenido como referentes teóricos el paradigma de la NGP y el enfoque 74 del capital humano. Este último se origina en el conductismo y en el pragmatismo 
representado por Barnard y Lewin. En ese sentido, en esta teoría se establece la visión gerencial desde una dimensión de eficiencia, eficacia y competitividad. Considera que el crecimiento económico es igual al desarrollo humano, como se señala en Bowles y Gintis (2014). Al igual que Fuertes, Plou y Gómez (2017), afirman que esta corriente ideológica basa el enfoque desarrollista en el producto interno bruto (PIB), que mide el desarrollo de un país. Los principios del capital humano privilegian el crecimiento económico; las personas son consideradas como cosas o como parte de las organizaciones; el desarrollo de la ciencia y los avances tecnológicos se priorizan en lugar de los seres humanos; estos últimos están al servicio del capital. En esta visión de la gerencia se manipula la actuación de las personas; la cultura es relegada; predomina la eficiencia, eficacia y competitividad a expensas de lo humano o lo social; el trabajador es únicamente la herramienta para la producción y la sociedad o el público un elemento de consumo (Polo, 2012 y Canto, 2011). Por su parte, el pragmatismo, de acuerdo con Barrena (2014, p. 1):

Es una corriente filosófica iniciada a finales del siglo XIX a raíz de la denominada máxima pragmática, propuesta por el lógico y científico norteamericano Charles $\mathrm{S}$. Peirce. Lejos de acepciones coloquiales y de interpretaciones erróneas, que ponen el énfasis en lo útil o en lo práctico, el pragmatismo original propugna que la validez de cualquier concepto debe basarse en los efectos experimentales del mismo, en sus consecuencias para la conducta.

Los representantes principales de la filosofía pragmática que han influido en América y en Europa son Charles S. Peirce, William James y John Dewey. Sus ideas y modo de ver la realidad, la verdad, las acciones y las repercusiones en la vida de los seres humanos se han extendido a diferentes áreas del conocimiento y las disciplinas. Sin embargo, existen actualmente representantes de esta corriente de pensamiento, como el norteamericano Richard Rorty, que defiende la idea de un pragmatismo con base en los criterios de la vida individual; otros como Hilary Putnam, que se afirman en un pragmatismo que permite al ser humano la libre elección de lo que quiere hacer con su vida y cómo quiere vivirla; esta noción también estaba presente en los filósofos que dieron origen a esta corriente (Barrena, 2014).

Por su parte, Stanley Fish cree en un pragmatismo basado en los valores de la comunidad. Nelson Goodman afirmaba que la realidad en el pragmatismo puede ser moldeable. Sus representantes llegaron a percibir y a entender que el pragmatismo es un conjunto de ideas, conceptos y creencias de la manera en que el hombre se encamina hacia la búsqueda de lo desconocido; comprendieron que el mundo es una realidad por descubrir que no está acabado, sino que el hombre tiene en la experiencia $\mathrm{y}$ en las acciones un potencial para el progreso de la humanidad si se actúa de una manera categórica (Barrena, 2014).

\section{El parradigamia de la nuevia gestión públicia}

La NGP tiene sus raíces en dos líneas ideológicas: la teoría de la elección pública y el gerencialismo. La primera es la que intenta exponer y advertir las conductas de los 
sujetos que intervienen en la política. La NGP retoma de esta teoría aquellos elementos en los que ya no cree que son capaces de contribuir al encargo establecido para mejorar los servicios públicos, como la centralización burocrática y la planeación, porque son insuficientes para tomar decisiones y contribuyen a errores y fracasos. En su lugar los sustituye por aspectos inherentes al mercado para contrarrestar el centralismo y la autosatisfacción de los trabajadores del gobierno; establece un conjunto de elementos, como los premios por el rendimiento y la productividad. Esta teoría alega que los funcionarios públicos actúan bajo el supuesto de que se aprovechan de su puesto para obtener rendimientos para sus propios bolsillos y se olvidan del compromiso que adquirieron para servir a los ciudadanos. También echó mano del gerencialismo e hizo suyas la necesidad de mayor libertad de acción para la dirección y administración de los asuntos públicos; partió del supuesto de que era necesario aligerar la carga burocrática liberando al sector público de limitaciones y leyes que inmovilizaban la prestación de los servicios, provocando el descontento de los ciudadanos. El propósito principal consistió en transformar o buscar mejores soluciones $\mathrm{y}$ acciones de formación, aunque se dieran errores en el proceso (Cejudo, 2013).

Otro componente del gerencialismo que formó parte de la NGP fue que el gobierno se ocupara mayormente de la dirección, desestimando actividades rutinarias y concentrándose en aquellas funciones enfocadas en la coordinación de las actividades administrativas. Del mismo modo, se apropió de conceptos originados en el mundo de la administración privada y de los negocios, como la inquietud por la mejora, la disposición a considerar al ciudadano como el comprador/consumidor, a medir los resultados como elemento de vigilancia y el deseo por el descubrimiento y la generación de valor público (Cejudo, 2013). Estos pasaron a formar parte del nuevo vocabulario de la vida en la administración pública de los países que adoptaron este nuevo enfoque para mejorar la prestación de los servicios, pero también para captar la voluntad y aprobación de los electores que bajo este esquema se volvieron más vigilantes de lo público y empezaron a exigir cuentas a sus gobernantes. Por su parte, organismos como el BM, la OCDE y el Centro Latinoamericano de Administración para el Desarrollo (CLAD) optaron por hacer suyos los postulados de la NGP y empezaron a recomendar a los países que hicieran efectiva la nueva orientación a fin de revitalizar todas las áreas de la administración pública.

\section{La nuevia gessión pública (IGGP) en Vévico}

Uno de los niveles gubernamentales influido por la NGP fue el sector educativo. En el caso de México, ha tenido poca incidencia en lo relacionado con la lógica del mercado y se ha enfocado más en la línea del gerencialismo, aunque esta ha sido muy frugal en lo referente a proporcionar mayor libertad a las áreas y al personal; del mismo modo se ha hecho poco para brindar pagos por mejor desempeño y productividad al personal.

A partir de los años ochenta, las reformas han constituido un parteaguas en políticas públicas con las llamadas reformas de primera, segunda y tercera generación. Las de primera generación tuvieron como objetivo estabilizar la economía de los 76 países financiados por los organismos internacionales e impulsar su crecimiento, to 
que implicó el desmantelamiento del Estado benefactor y se dejó paso a la inversión privada nacional e internacional y al libre mercado. En esta década, México aceleró su ingreso al Acuerdo General sobre Comercio y Aranceles (GATT, por sus siglas en inglés) y se firmó el Tratado de Libre Comercio con América del Norte (TLCAN); las reformas de primera generación, en materia de política educativa significaron la descentralización y la reducción en el financiamiento de la educación (Del Castillo-alemán, 2012; Trejo y Andrade, 2013).

También se caracterizaron por el establecimiento, desarrollo y ejecución de políticas públicas en México con base en acuerdos con estos organismos internacionales derivado de la crisis económica que se suscitó en los años setenta con motivo de la caída de los precios del petróleo y el aumento en las tasas de interés de la deuda externa que tuvo repercusiones en las décadas siguientes. El entonces presidente de la república Miguel de la Madrid Hurtado (1982-1988) acudió a la comunidad financiera internacional para reestructurar las condiciones y términos de la deuda a fin de no caer en una moratoria de pagos de manera unilateral. Los organismos internacionales respondieron con prontitud en apoyo de México y suscribieron una carta de intención en la que el gobierno se comprometió a una serie de medidas que ayudaron en la estabilidad económica, pero surgieron efectos negativos que impactaron en el desempleo, en el ingreso per cápita de la población y en el gasto social, lo que afectó las partidas presupuestales de las áreas de salud y educación (Alcántara, 2008).

El 18 de mayo de 1994, México se convirtió en miembro de la OCDE; el decreto fue publicado en el Diario Oficial de la Federación el 5 de julio del mismo año. En él se establece que México se adhiere, entre otras, a la Declaración sobre Políticas Educativas Futuras en el Contexto Económico y Social de Cambio de fecha 20 de octubre de 1978 (Secretaría de Gobernación, 1994).

Por su parte, las reformas de segunda generación tuvieron como objetivo la profundización de las reformas de primera generación, como fue el caso del proceso de apertura comercial, una mayor participación de la inversión extranjera directa y se aceleró el mecanismo que impulsó la competitividad internacional, la inversión y el crecimiento económico. En lo referente a la orientación de políticas educativas, se tuvo como propósito la evaluación del sistema educativo (Trejo y Andrade, 2013; Del Castillo-Alemán, 2012).

Finalmente, las reformas de tercera generación fueron dirigidas a la reforma laboral, fiscal, energética y educativa (Trejo y Andrade, 2013). En relación con las políticas educativas se modificó el artículo tercero constitucional y la Ley General de Educación (LGE), se crearon la Ley del Servicio Profesional Docente (LSPD) y la Ley del Instituto Nacional para la Evaluación de la Educación (INEE) dotándolo de autonomía.

Los acuerdos con estos organismos financieros y de cooperación internacional incidieron en la política pública nacional en todos los órdenes de gobierno. El sistema educativo no fue la excepción. Las reformas tocaron fibras muy sensibles en la sociedad mexicana, como la relacionada con la educación pública y gratuita, la descentralización educativa, la reducción del gasto social -en el que se incluye a la educación-, la reducción de la matrícula de ingreso a la educación superior y la exi- 
gencia de una mayor eficacia y eficiencia en las instituciones de educación superior. La conexión de lo anterior con las políticas laborales y las exigencias de formación de profesionales altamente competitivos en todas las ramas del conocimiento son un tópico que está en el centro del debate internacional.

Las políticas que inciden en el sistema educativo se ven reflejadas, en el caso de México, en el nivel de educación básica que comprende tres años de preescolar, seis años de primaria y tres de secundaria. El nivel medio superior comprende tres años y el nivel de educación superior varía entre cuatro y cinco años. El grueso de la población estudiantil se encuentra en el nivel básico. Los profesionistas que forman a los estudiantes de este nivel son egresados de las escuelas normales.

\section{La políticia públicia de formación de maestros en México}

La política de formación de maestros en México inició en el año de 1906 en que se creó la Ley Constitutiva de las Escuelas Normales. Antes de este periodo, las exigencias para ser maestro eran mínimas. Las Escuelas Lancasterianas, fundadas en 1822, eran las instituciones que autorizaban el ejercicio de la profesión; posteriormente esta función pasó a manos del gobierno municipal y de ahí a la federación, lo que convirtió a la docencia en una profesión de Estado. En 1911, con Justo Sierra, se dio un gran impulso a la Escuela Normal y de Altos Estudios creada en 1902. En 1922 se creó en Michoacán la primera escuela normal rural. En 1924, al interior de la Universidad Nacional nació la Escuela Normal Superior con la intención de formar profesores que trabajaran en escuelas secundarias, normales, profesionales y fungieran como directores de escuelas (Navarrete-Cazalez, 2015).

Con José Vasconcelos al frente de la Secretaría de Educación Pública (SEP) en 1921, el establecimiento de las políticas públicas educativas en México recibió un fuerte impulso debido a que durante su gestión se consideró que estas serían un instrumento para que el país se desarrollara tanto cultural como democráticamente (Galván, 2016). En México, la SEP es la responsable de conducir y establecer las directrices del desarrollo educativo del país. La desconcentración de la propia SEP en 1978 constituyó uno de los antecedentes más importantes en materia de política educativa (Gómez, 2017).

Los educadores que forman a los estudiantes del nivel básico son egresados de las escuelas normales. Estas instituciones son las encargadas de la formación de docentes de educación básica desde su creación a finales del siglo XIX (Navarrete-Cazalez, 2015). Entre los objetivos de las primeras escuelas normales, de acuerdo con Arnaut (1998, p. 26), estaba el más importante de ellos:

Formar maestros y autorizar el ejercicio de la docencia, así como el de integrar un grupo de profesionistas con características muy específicas que se equiparara en rango al de otros grupos de profesionistas para superar el empirismo de los maestros egresados 0 reclutados por distintos medios diferentes a las Normales y, finalmente, dar unidad y uniformidad técnica o científica a la enseñanza primaria liberando la profesión docente y a las escuelas de la influencia que agentes externos ejercían sobre la profesión. 
Durante el periodo de José Vasconcelos Calderón al frente de la SEP, la política de formación de maestros se enfocó en la alfabetización y en la educación rural. En 1925, la Escuela Normal de Profesores de Instrucción Primaria, creada en 1887, se convirtió en la Escuela Nacional de Maestros y ahí se formaba, primero durante tres años y posteriormente seis años, a los profesores en la corriente de la escuela activa, incluyendo los tres años de la secundaria y asumiendo la coeducación. En 1935, la formación de maestros transita del socialismo a la influencia del pragmatismo y el neopositivismo, cuya tendencia es hacia el modelo desarrollista por medio de la ciencia y la tecnología.

En 1942 se creó la Normal Superior de México, cuyo requisito para ingresar fue el bachillerato o los estudios de la normal básica. La formación de maestros se orientó hacia un movimiento cultural y artístico, sin descuidar la ciencia y la formación de un técnico de la educación. Luego se creó la Dirección General de Enseñanza Normal. Debido al desmedido crecimiento de estas instituciones, el funcionamiento y organización se fue haciendo más complejo. En 1954 se creó la Junta Nacional de Educación Normal con el fin de revisar los perfiles de los formadores de docentes. De 1958 a 1970, la política educativa continuó con el Plan de Once Años, en cuyo propósito estaba el de atender a la población estudiantil de 6 a 14 años de edad, la creación de plazas docentes y en la búsqueda de la eficiencia terminal en al menos el 38\%. En 1960 se crearon los Centros Regionales de Educación Normal (CREN) con un nuevo plan de estudios con el fin de mejorar la calidad de la formación docente (Navarrete-Cazalez, 2015).

En 1969 se llevó a cabo una reforma a los planes de estudio de las escuelas normales, separándose la enseñanza secundaria de los estudios de las normales y el periodo de permanencia se amplió a cuatro años. Para 1970, en la SEP se crearon cuatro secretarías; una de ellas fue la Secretaría de Educación Primaria y Normal. En el año de 1984, la Dirección General de Educación Normal (DGEN) dependía de la Subsecretaría de Educación Superior e Investigación Científica (SESIC). Durante el periodo al que se ha hecho referencia, la educación normal experimentó siete reformas; en estas destaca aquella que permitió a los normalistas obtener al mismo tiempo el título de profesor de educación primaria y el certificado de bachillerato. En la década de los ochenta los enfoques teóricos que asistieron a la modernización de la educación superior fueron la teoría del capital humano y el marxismo. Una muestra de ellos es que en 1984 se produjo la siguiente reforma a la educación normal que estableció como requisito los estudios de bachillerato para estudiar en las escuelas normales. Este hecho significó un cambio estructural en estas instituciones para el cual no estaban preparadas y cuyas consecuencias aún se resienten (Navarrete-Cazalez, 2015).

\section{Vì́rouno}

Mediante una búsqueda sistemática se revisaron artículos científicos, revistas indexadas, libros de administración pública y políticas públicas educativas en educación superior en las bases de datos de Google Scholar, Dialnet, Redalyc, SciELO y La- 
tindex. El periodo de 1984 a 2018 se delimitó con base en que a partir de 1984, los estudios de las escuelas normales (EN) en México se elevaron a nivel de licenciatura; en esa fecha se les empezó a considerar entre el grupo de instituciones de educación superior (IES) y posteriormente en 1996 el gobierno, a través de la Secretaría de Educación Pública (SEP), inició el diseño de políticas para profesionalizar a los formadores de docentes que laboran en las EN con el propósito de elevar la calidad de la formación inicial de los profesores de educación básica y sometió a estas instituciones a la evaluación interna y externa.

Para la estrategia de búsqueda se usaron los términos: políticas educativas de educación normal, profesionalización docente en escuelas normales en México, el paradigma de la NGP y características de la NGP. Se seleccionaron artículos de revisión de la literatura, artículos con evidencia empírica, libros de editoriales internacionales reconocidas, informes de organismos nacionales e internacionales; se eligieron textos de fuentes primarias. Los artículos que se seleccionaron cumplieron con los criterios de inclusión que se establecieron para la búsqueda.

Las categorías que se crearon para el análisis de cada texto fueron: organismos internacionales y nueva gestión pública, políticas educativas de educación superior en educación normal, profesionalización docente de escuelas normales en México. Se utilizó una lectura crítica de acuerdo con los siguientes criterios: características del artículo, se constató que se tratara de la literatura que se había contemplado, revisión de la metodología de la investigación, análisis de los resultados, discusión e implicaciones, conclusiones y comentarios. Para la revisión de la literatura se llevó a cabo el siguiente proceso: se leyó el nombre de los autores, se revisó el año en que fue escrito, se interpretó el título del artículo, se identificaron las secciones en que se dividió, se observaron los gráficos, figuras, tablas, fotos y finalmente se revisó la bibliografía para asegurarse que fuera reciente 0 estuviera relacionada con el tema.

Los criterios de inclusión fueron artículos en revistas arbitradas en línea y con texto publicadas entre 1984 y 2018; se priorizaron estudios cualitativos que explícitamente mencionaran los conceptos de políticas educativas de educación normal en México, profesionalización docente en escuelas normales y que fuera posible extraer información relevante; políticas educativas de educación superior y el paradigma de la NGP y características de la NGP. Los criterios de exclusión fueron artículos escritos antes de 1984, textos con políticas educativas de educación superior universitaria, artículos con investigaciones de educación normal de otros países (Kitchenham, 2004; Caro et al., 2005; Garcés y Duque, 2007; Díaz, 2009; Rudas, Gómez y Toro, 2013; García, 2017; y Ribes, Perelló y Pantoja, 2017). Se utilizó el método comparativo, el cual describe similitudes y disimilitudes a partir del presente y se despliega de forma horizontal, confronta objetos que conciernen a igual género basado en el razonamiento de uniformidad y por lo tanto difiere de la sola comparación. Este análisis se basó en la búsqueda de similitudes de las políticas de profesionalización docente que se han establecido en las EN de México en relación con los principios de la NGP (Tonón, 2011; Sartori, 1994). 


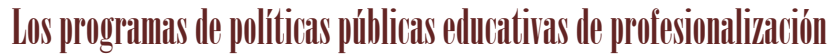 de miestiros en las escuelas normilles de lévico}

De acuerdo con Arnaut (1998), la reforma de los planes y programas de las escuelas normales de 1984 tuvo dentro de sus objetivos el de reorganizar la enseñanza normal para cumplir mejor con sus fines, fortalecer la coordinación y la evaluación continua, regular la matrícula, mejorar la calidad de la enseñanza y satisfacer las necesidades de formación del magisterio mediante programas de actualización y superación docente, además de estimular los esfuerzos académicos del maestro, propiciar la profesionalización de la docencia y fortalecer las funciones de docencia, investigación y difusión cultural de las escuelas normales como verdaderas IES.

Esta reforma dejó sumido a los formadores de docentes en la indefinición e incertidumbre de lo que debía ser la formación de maestros, ya que agregó más responsabilidades a los académicos para las que no estaban preparados; implicó que los formadores se constituyeran como docentes reflexivos y profesores-investigadores, funciones para las que no habían sido formados o actualizados (Navarrete-Cazales, 2015). Aun así, los procesos de reforma continuaron y con la intención de subsanar las deficiencias de los formadores de docentes se fueron estableciendo políticas que contribuyeran a su profesionalización. A continuación, se describen los programas de políticas públicas educativas de profesionalización docente de las escuelas normales en México.

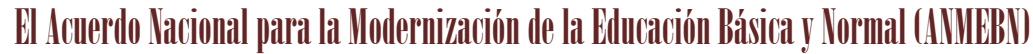

De acuerdo con Zorrilla (2002, p. 2), la firma de este acuerdo el 18 de mayo de 1992:

Es el acontecimiento detonador de un amplio proceso de reforma de la educación básica y de la formación de maestros. Este hecho no es aislado, se inserta en un contexto más amplio de la Reforma del Estado mexicano y significa uno de los pactos políticos más importantes del siglo veinte al plantear decisiones sobre dos asuntos fundamentales para el desarrollo futuro del Sistema Educativo Mexicano: la descentralización del sistema educativo y una reforma curricular y pedagógica para la educación básica obligatoria y la formación inicial de maestros.

Las políticas públicas que dieron origen al proceso de reforma que se inició con la firma de este acuerdo han hecho énfasis en que el Estado debe dejar de ser un administrador y convertirse en moderador de los procesos y cambios innovadores de la gestión del Estado. La función de la escuela hasta antes del acuerdo era la de formar a los estudiantes; después del acuerdo esa perspectiva ha cambiado y ahora la mirada es diferente, porque se pretendió que la escuela fuera el centro del sistema educativo con autonomía, con la capacidad para gestionar sus propios recursos que satisfagan las necesidades de profesionalización y sometida al escrutinio público, además de la formación de los alumnos. 


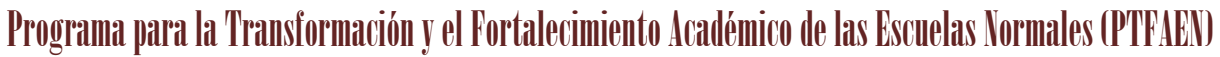

Fue creado en 1996. De acuerdo con la SEP (2003a, pp. 9, 11), el PTFAEN estableció originalmente las siguientes líneas de trabajo: "transformación curricular; actualización y perfeccionamiento profesional del personal docente; elaboración de normas y orientaciones para la gestión institucional y la regulación del trabajo académico; y mejoramiento de la planta física y del equipamiento de las escuelas normales".

Posteriormente, este programa replanteó sus acciones y agregó más líneas que según la SEP (2003a, p. 11) fueron: "[...] transformación curricular, formación y actualización de maestros y directivos, mejoramiento de la gestión institucional, regulación del trabajo académico, evaluación interna y externa, y regulación de los servicios que se ofrecen de educación normal".

Se replanteó la necesidad e importancia del trabajo colegiado de los docentes. Posteriormente, un año después, la Dirección General de Normatividad de la Subsecretaría de Educación Básica y Normal publicó un documento en el que enfatizaba la importancia del trabajo colegiado, puntualizando sugerencias para su organización y las tareas que cada colectivo debía realizar; sin embargo, los profesores solo llegaron al nivel de conocer el documento, pero no hicieron suyas las propuestas de la SEP (Espinosa, 2008).

Ahora bien, del PTFAEN se derivó la reforma de 1997 para la licenciatura en educación primaria y la de 1999 para la licenciatura en educación preescolar y educación secundaria, en el 2002 para la licenciatura en educación física y en el 2004 para las licenciaturas en educación especial y primaria con enfoque intercultural bilingüe.

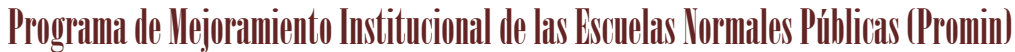

De acuerdo con la SEP (2003a, p. 13):

El PROMIN constituye una respuesta para continuar estimulando la transformación en la organización y funcionamiento de las escuelas normales. Busca generar condiciones institucionales propicias para el mejoramiento de la formación inicial de los maestros de educación básica y la profesionalización de sus maestros, de acuerdo con las orientaciones establecidas en los planes y programas de estudio.

Se propuso lograr lo anterior mediante la mejora de la planta física, el equipamiento y las acciones de trabajo conjunto con directivos, personal de apoyo, docentes y alumnos en las instituciones de educación normal. Igualmente se buscaba que las escuelas normales se constituyeran en grupos de aprendizaje lo suficientemente cohesionados con la finalidad de mejorar la formación inicial de maestros en estas instituciones. Este programa contempló, de acuerdo con la SEP (2003b, p. 22):

La construcción de una nueva propuesta basada en la profesionalización de la enseñanza, considera la formación docente como un proceso integral de fases y ámbitos interrelacionados: admitir en la carrera a los estudiantes potencialmente más capaces; ofrecer una sólida formación inicial; contratar a quienes demuestren las competencias básicas 
para el ejercicio docente, y estimular y retener los docentes eficaces en la profesión, continuando su formación y desarrollo profesional. Lo anterior, debe vincularse con el mejoramiento de las condiciones para la enseñanza y el aprendizaje, con incentivos que motiven a los maestros a asumir la profesión con entusiasmo, preparación suficiente y responsabilidad, así como con mecanismos sistemáticos de evaluación de dichas fases y ámbitos.

Este programa pretendió que las escuelas normales del país poco a poco fueran adquiriendo una mayor autonomía con base en dos elementos de planeación; el plan de desarrollo institucional (PDI) y el programa anual de trabajo (PAT), característica fundamental que les ayudaría a conformarse como una verdadera IES.

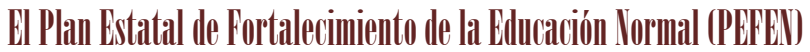

Se estableció en 2005. En concordancia con la SEP (2006), el PEFEN buscaba integrar y consolidar la eficacia de las instituciones formadoras de docentes, así como contribuir en la mejora de la prestación educativa y en el trabajo conjunto de directivos, docentes, personal de apoyo y alumnos. La puesta en marcha de este plan pretendía que estas instituciones se transformaran y se establecieran sólidamente como verdaderas IES, integrándose plenamente como tales. Lo anterior aspiraba a reducir las diferencias de calidad. En este sentido, representa una oportunidad para reducir los huecos en la eficacia de la formación de docentes entre los estados; del mismo modo, establecer poco a poco la cultura del escrutinio público sobre los resultados y la evaluación del presupuesto destinado a la educación de este nivel.

Este programa se auxilia de dos herramientas que contribuyen a lograr su concreción; uno de ellos es el Programa de Gestión de la Educación Normal (Progen). En este se detallan las acciones y políticas que se establecen desde la entidad federativa y el Programa de Fortalecimiento de la EN (Profen), en el cual se especifican las acciones concretas que se desarrollan en cada EN.

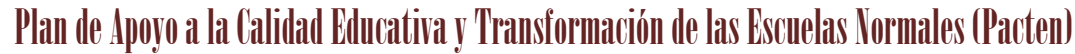

Para el año 2014, el PEFEN se transformó en el Pacten, manteniendo los mismos propósitos y los programas que lo concretizan en el nivel de la entidad federativa y en el de las EN. Ahora bien, a partir de que las EN pasaron a depender de la Subsecretaría de Educación Superior en el año 2005, se creó la Dirección General de Educación Superior para Profesionales de la Educación (DGESPE) para impulsar el mejoramiento del subsistema de educación normal y continuar el proceso de innovación y afianzamiento de estas instituciones como auténticos organismos que ofrecen educación superior de alta calidad. Para este fin, en el año 2008 las escuelas normales iniciaron el camino de la evaluación externa a través de los Comités Interinstitucionales para la Evaluación de la Educación Superior (CIEES); este modelo de evaluación se había establecido con anterioridad para las universidades desde el año de 1992. 


\section{Programia parra el Desirirollo Profesional Inocente (Prodep)}

Este se diseñó originalmente como Programa para el Mejoramiento del Profesorado (Promep) en 1996 para las universidades; posteriormente se transformó en el Programa para el Desarrollo Profesional Docente de tipo superior (Prodep). Sus objetivos son que el personal docente de tiempo completo alcance la máxima habilitación en estudios de posgrado y la conformación de cuerpos académicos. Está basado en que el personal académico equilibre, en la medida de lo posible, las funciones de docencia, la gestión académica, la tutoría y la generación de conocimiento; busca que estas cuatro funciones se desarrollen con el mismo nivel de competencia. Su fin último es promover una educación superior de buena calidad. El instrumento para medirla es la evaluación de las acciones, los resultados y la productividad con base en los métodos de la NGP, la cual tiene implícitas las características de control del mercado que impulsó a las universidades bajo las fuerzas de la competencia entre ellas por la asignación de los recursos (Cordero et al., 2009; Pérez, 2013). Para el año 2009, las escuelas normales fueron incluidas en este programa con el mismo esquema de las universidades.

\section{Conculsiones}

La revisión de la literatura da cuenta del diseño, establecimiento, ejecución y evaluación de las políticas de profesionalización de maestros en México durante el periodo revisado, que va de 1984 a 2018. Estas políticas se han establecido desde la teoría del capital humano con referentes como el conductismo y el pragmatismo y los postulados de la nueva gestión pública, que a su vez retoma la teoría de la elección pública que hace énfasis en el enfoque del mercado y la teoría del gerencialismo, que enfatiza o privilegia la dirección y relega los aspectos rutinarios de la administración, dejando una mayor libertad de maniobra al personal involucrado. La conducción de estas políticas se ha hecho con la recomendación y la asesoría de organismos internacionales como el BM, el BID, la OCDE y la UNESCO (por sus siglas en inglés) (García-Sánchez, 2017). En este sentido, se encontró que el establecimiento y ejecución de estos enfoques teóricos no son necesariamente homogéneos, especialmente en los países de América Latina. En el caso de México se ha preferido la teoría del gerencialismo en las políticas que sobre el sector educativo se han llevado a cabo. Se halló que, a lo largo de este tiempo, como parte de esta política, se han establecido cinco programas: cuatro diseñados completamente nuevos y uno reestructurado, que han tenido como propósito fundamental fortalecer la formación de los formadores de docentes en las escuelas normales.

Sin embargo, los resultados del establecimiento de estas políticas de profesionalización docente, alineadas con la profesionalización en las universidades, refleja la disparidad de las nuevas reglas y la desventaja en la que se encuentran las escuelas normales, porque estas instituciones, desde su creación, tienen una amplia trayectoria enfocada en la formación de docentes y poca inclinación hacia la investigación y la 84 difusión del conocimiento, como lo señala un artículo publicado por Edel-Navarro, 
Ferra-Torres y de Vries (2018). Una de las razones principales, afirman los autores, para que el Prodep, a casi diez años de su puesta en marcha, tenga tan pocos resultados en el propósito de encauzar las acciones sustantivas de las escuelas normales en el mismo camino de las universidades públicas; es la ventaja que las universidades tienen en relación con la amplia experiencia que poseen para la investigación, por lo que se requieren cambios estructurales en el que los actores de las escuelas normales se sientan incluidos, para que estas instituciones funcionen, al menos, de modo parecido a las universidades.

Ahora bien, esta política ha tenido como ejes el trabajo conjunto de directivos, docentes, alumnos y personal de apoyo. La planeación institucional tiene un enfoque transcendental y se basa en una serie de estudios que tienen la finalidad de anticiparse a los sucesos que pueden ocurrir de manera futura; los demás ejes apuntan a la autonomía de las escuelas normales, la capacitación y actualización de los profesores, la evaluación interna y externa, la construcción y el equipamiento de la planta física y la infraestructura.

La revisión que se hizo de los documentos demuestra la similitud existente en el diseño de las políticas públicas sobre educación superior para las escuelas normales. Las acciones se han orientado por los principios de la NGP. En una investigación de Gutiérrez (2006), se encontró que en estos programas que se instauraron con este tipo de políticas públicas el gobierno privilegia la supervisión de las acciones y se desentiende del abastecimiento de los recursos a las escuelas normales, sometiéndolas a la dinámica de la competencia por el presupuesto. En relación con la profesionalización docente en las escuelas normales es claro que las recomendaciones de los organismos internacionales son notorias; así lo señala Ortega (2014) al afirmar que la elaboración de la planeación de las escuelas normales, que les permite concursar por los recursos, tiene claramente una orientación que llega desde las recomendaciones de estas organizaciones que condicionan el financiamiento al cumplimiento de las políticas públicas para afianzar a las instituciones formadoras de docentes como verdaderas instituciones de educación superior, con profesores que tengan altas competencias e idoneidad académica, capaces de establecer procesos transformadores de gestión de las instituciones y del conocimiento.

Por su parte, otros autores, como Zorrilla (2002), hacen referencia a las políticas públicas educativas instauradas en México desde la perspectiva de las reformas internacionales, sin hacer referencia directa. No obstante, los postulados de la NGP están claramente representados en la amplia gama de propuestas que han dado origen a los cambios estructurales en la profesionalización de maestros de las escuelas normales.

Los resultados de las políticas públicas educativas en México, orientadas desde el plano internacional, han sido muy diversos, debatidos por voces a favor y en contra; sin embargo, se han ido abriendo camino en medio de la crítica, la oposición o el beneplácito de los diversos grupos de la sociedad mexicana. Así lo señala un artículo publicado en México por Guerrero y Huitzil (2014), en el que hace un amplio recuento de lo sucedido en este país después de diez años del establecimiento de las políticas educativas de profesionalización docente. Alude a que las políticas han incidido prioritariamente en la cantidad y se ha soslayado la calidad y la equidad; no obstante, elogia la capacidad del gobierno para aglutinar a las fuerzas políticas a favor de uno 
de los movimientos de transformación estructural más grande del siglo pasado. Por el contrario, otro artículo, también publicado en México por Sánchez y Corte (2015), manifiesta que las políticas educativas de profesionalización de maestros solo son una reforma laboral que va en contra de los derechos de los profesores en México y que dichas reformas se diseñan desde los organismos internacionales, provocando cambios estructurales en las leyes y reglamentos de los países miembros, como en el caso de México que las políticas han obligado a modificar la Constitución y crear nuevas leyes como la Ley General del Servicio Profesional Docente, así como la creación de instancias reguladoras y evaluadoras autónomas como el Instituto Nacional para la Evaluación de la Educación (INEE).

La revisión de la literatura sobre la profesionalización docente en las escuelas normales muestra un abanico de posiciones respecto de las políticas públicas educativas desde distintos enfoques y corrientes teóricas de los actores, en el que se refleja la diversidad ideológica de los profesores y sus perspectivas sobre las escuelas normales, quienes, sin duda, deberán encontrar su propio sitio para reorganizarse, evolucionar y seguir formando a los maestros de México acordes con la visión que requiere la sociedad del siglo XXI, sin perder la identidad que les dio origen. Lo anterior constituiría una nueva línea de investigación de la que, a partir de las voces de los actores, se establezca el rumbo de estas instituciones como formadoras de docentes o como instituciones de educación superior, con sus propias leyes y reglamentos 0 con las mismas reglas de la educación superior universitaria.

\section{Referencitis}

Arnaut, A. (1998). Historia de una profesión. Los maestros de educación primaria en México, 1887-1994. México: Subsecretaría de Educación Básica y Normal / SEP.

Alcántara, A. (2008). Políticas educativas y neoliberalismo en México: 1982-2006. Revista Iberoamérica de Educación, (048), 147-165. Organización de Estados Americanos para la Educación, la Ciencia y la Cultura. Recuperado de https://www.researchgate.net/profile/ Armando_Alcantara2/publication/237040258_Politicas_educativas_y_neoliberalismo_en_ Mexico_19822006/links/551afd490cf2bb754078799f.pdf

Barzelay, M. (2013). La nueva gestión pública. En G. Cejudo (comp.), Nueva gestión pública (col. Biblioteca básica de administración pública n. 10, pp. 114-158). México: Siglo XXI. Recuperado de http://www2.df.gob.mx/virtual/evaluadf/docs/estudios/i_ngp_eap.pdf

Barrena, S. (2014). El pragmatismo. Factótum, (12), 1-18. Recuperado de http://www. revistafactotum.com/revista/f_12/articulos/Factotum_12_1_Sara_Barrena.pdf

Bowles. S. y Gintis, H. (2014). El problema de la teoría del capital humano. Una crítica marxista. Revista de Economía Crítica, (18), 220-228. Recuperado de www.revistaeconomiacritica. org/.../15_Bowles-Gintis_Teoria-del-capital-humano.pdf

Caro, M., Rodríguez, A., Calero, C., Fernández-Medina, E. y Pialtini, M. (2005). Análisis y revisión de la literatura en el contexto de proyectos de fin de carrera: una propuesta. Sociedad Chilena de Ciencia de la Computación, 6(1), 1-9. Recuperado de https://users.dcc.uchile. $\mathrm{cl} / \sim \mathrm{mmarin} /$ revista-sccc/sccc-web/Vol6/CCESC08.pdf

Canto del E. (2011). Gestión del capital humano, competencias y sociedad del conocimiento. Observatorio Laboral, 4(8), 89-113. Recuperado de https://www.redalyc.org/articulo. oa?id=219022148006 
Cejudo, M.G. (2013). Nueva gestión pública (col. Biblioteca básica de administración pública n. 10). México: Siglo XXI. Recuperado de http://www2.df.gob.mx/virtual/evaluadf/docs/ estudios/i_ngp_eap.pdf

Chica, V.S. (2011). Una mirada a los nuevos enfoques de la gestión pública. Administración \& Desarrollo, 39(53), 57-74. Recuperado de https://dialnet.unirioja.es/descarga/ articulo/3776682.pdf

Cordero, A.G., García, G.J., Galaz F.J., Nishikawa, A.K. y Antillón, M.L. (2009). Publicación científica y evaluación docente en México: un diagnóstico e intervención con profesores de educación y humanidades de una universidad estatal. Tiempo de Educar, 10(19), 149-168. Recuperado de http://www.redalyc.org/pdf/311/31113164006.pdf

del Castillo-Alemán, G. (2012). Las políticas educativas en México desde una perspectiva de política pública: gobernabilidad y gobernanza. Revista Internacional de Investigación en Educación, 4(9), 637-652. Recuperado de www.redalyc.org/pdf/2810/281022848007.pdf

Díaz, J. (2009). Guía práctica de lectura crítica de artículos científicos originales en ciencias de la salud. Madrid: Instituto Nacional de Gestión Sanitaria. Recuperado de http://www.ingesa. mscbs.gob.es/estadEstudios/documPublica/internet/pdf/Guia_practica_de_lectura.pdf

Edel-Navarro, R., Ferra-Torres, G. y de Vries, W. (2018). El Prodep en las escuelas normales mexicanas: efectos y prospectiva. Revista de la Educación Superior, 47(187). Recuperado de resu.anuies.mx/ojs/index.php/resu/article/download/419/257/

Espinosa, M.E. (2008). El trabajo colegiado en las escuelas normales y la evaluación de sus planes de estudio. CPU-e. Revista de Investigación Educativa, (7). Recuperado de http://www. uv.mx/cpue/num7/opinion/espinosa trabajo_colegiado.html

Fuertes, G.E., Plou L.P. y Gómez, C. (2017). Desarrollo humano desde la perspectiva del crecimiento. Revista de Ciencias Sociales, (4), 81-97. Recuperado de https://www.redalyc. org/articulo.oa?id=28055641007

García, P.F.J. (2017). Revisión sistemática de literatura en los trabajos de final de máster y en las tesis doctorales. Salamanca, España: Programa de Doctorado Formación en la Sociedad del Conocimiento / Facultad de Ciencias. Recuperado de https://knowledgesociety.usal.es/sites/ default/files/20170316\%20-\%20Seminario\%20SLR.pdf

García-Sánchez, L. (2017). La investigación educativa como elemento fundamental para el desarrollo académico de una escuela normal como institución de educación superior: una mirada desde la planeación estratégica en el Profen. Ra Ximhai, 13(3), 41-50. Recuperado de https://www.redalyc.org/articulo.oa?id=46154070002

Garcés, C.J. y Duque, O.E. (2007). Metodología para el análisis y la revisión crítica de artículos de investigación. INNOVAR. Revista de Ciencias Administrativas y Sociales, 17(29), 184-194. Recuperado de http://www.redalyc.org/articulo.oa?id=81802912

Galván, L.L.E. (2016). Derecho a la educación. Grandes temas constitucionales. México: Instituto Nacional de Estudios Históricos de las Revoluciones Mexicanas, Secretaría de Cultura. Recuperado de https://archivos.juridicas.unam.mx/www/bjv/libros/9/4450/13.pdf

Guerrero, C.J.M. y Huitzil, S.J.M. (2014). El Programa de Fortalecimiento de las Escuelas Normales (Profen): impacto en la capacidad académica de los docentes de la Licenciatura en Educación Preescolar del Benemérito Instituto Normal del Estado Gral. Juan Crisóstomo Bonilla. Revista Iberoamericana para la Investigación y el Desarrollo Educativo, (12), 1-19. Recuperado de ride.org.mx/1-11/index.php/RIDESECUNDARIO/article/download/761/743

Gómez, M.E. (2017). Panorama del sistema educativo mexicano desde la perspectiva de las políticas públicas. Innovación Educativa, 17(74), 143-163. Recuperado de http://www.scielo. org.mx/scielo.php?script=sci arttext\&pid=S1665-26732017000200143\&lng=es\&tlng=en

Gutiérrez, C. (2006). El mejoramiento institucional de las escuelas normales en el gobierno del cambio. Reencuentro. Análisis de Problemas Universitarios, (45). Recuperado de http://www. redalyc.org/pdf/340/34004506.pdf

Kitchenham, B. (2004). Procedures for performing systematic reviews (technical report TR/SE0401). Keele University. Recuperado de http://www.inf.ufsc.br/ aldo.vw/kitchenham.pdf 
Moreno, A.C.I. (2017). Las reformas en la educación superior pública en México: rupturas y continuidades. Revista de la Educación Superior, 46(182), 27-44. http://doi.org/10.1016/j. resu.2017.03.001

Navarrete-Cazales, Z. (2015). Formación de profesores en las escuelas normales de México. Siglo XX. Historia de la Educación Latinoamericana, 17(25), 17-34. http://doi. org $/ 10.19053 / 01227238.3805$

Organización de las Naciones Unidad para la Educación, la Ciencia y la Cultura (UNESCO). (1996). La educación encierra un tesoro. Informe a la UNESCO de la Comisión Internacional sobre la Educación para el siglo XXI. Recuperado de http://unesdoc.unesco.org/ images/0010/001095/109590so.pdf

Parcerisa, L. (2016). Nueva gestión pública y reforma educativa: la recontextualización de la autonomía escolar en diferentes contextos escolares en Cataluña. Revista Portuguesa de Educação, 29(2), 359-390. https://doi.org/10.21814/rpe.7928

Pérez, D.A. (2013). Políticas públicas y trabajo académico. Un referente internacional para la conducción de la política pública en México. Reencuentro. Análisis de Problemas Universitarios, (67), 26-40. Recuperado de https://www.redalyc.org/articulo. oa? id $=34030523004$

Polo, de L.G. (2012). Teoría organizacional para la gerencia humana como factor de desarrollo a escala humana. Clío América, 6(11). 52-71. Recuperado de https:/dialnet.unirioja.es/ descarga/articulo/5114830.pdf

Ramírez, C.C. y Jiménez, B.W.G. (2008). Gobierno y politicas públicas. Programa administración pública territorial. Módulo gobierno y políticas públicas. Bogotá, D.C.: Escuela Superior de Administración Pública. Recuperado de http://www.esap.edu.co/portal/wp-content/ uploads/2017/10/1-Gobierno-y-Politica-Publica.pdf

Ribes, G.G., Perelló, M.M.R. y Pantoja, D.O. (2017). Revisión sistemática de literatura de las variables clave del proceso de co-creación en las instituciones de educación superior. TEC Empresarial, 11(3). Recuperado de https://www.scielo.sa.cr/pdf/tec/v11n3/1659-3359tec-11-03-00041.pdf

Röth Deubel, A. (2003). Introducción para el análisis de las políticas públicas. Cuadernos de Administración, 19(30), 113-128. https://doi.org/10.25100/cdea.v19i30

Rudas, J.S., Gómez, L.M. y Toro, A.O. (2013). Revisión sistemática de literatura. Caso de estudio: modelamiento de un par deslizante con fines de predecir desgaste. Prospect, 11(1), 50-58. Recuperado de https://dialnet.unirioja.es/descarga/articulo/4697704.pdf

Sánchez, C.M. y Corte, C.F. del S. (2015). La OCDE, el Estado y los maestros de la CNTE: un estudio de la reciente reforma laboral. Revista Latinoamericana de Estudios Educativos, XLV(4), 121-156. México. Recuperado de https://www.redalyc. org/articulo.oa? id=27043549006

Sartori, G. (1994). Comparación y método comparativo. (Versión de Russo, J. y Ruíz de Azúa, M. A.) La comparazione nelle scienze social. (pp.14-29). Bologna. Sartori, G. y Morlino, L. (Comp.) Por Societá Editrice Il Mulino, Ed., 1991, Bologna. Recuperado de: https://www.academia.edu/9478926/La_comparacion_en_las Ciencias_Sociales_Giovanni_Sartoti

Secretaría de Gobernación (SG). (1994). Decreto de promulgación de la declaración del gobierno de los Estados Unidos Mexicanos sobre la aceptación de sus obligaciones como miembro de la Organización de Cooperación y Desarrollo Económicos. Diario Oficial de la Federación. Recuperado de http://dof.gob.mx/nota detalle. php? codigo $=4711014 \&$ fecha $=05 / 07 / 1994$

Secretaría de Educación Pública (SEP). (2003a). Programa de Mejoramiento Institucional de las Escuelas Normales Públicas (Promin). Programa para la Transformación y el Fortalecimiento de las Escuelas Normales. Finalidades, características y estrategias de operación. Serie Gestión Institucional 2. Dirección General de Normatividad. Subsecretaría de Educación Básica y Normal. México. Recuperado de https://www. dgespe.sep.gob.mx/public/ddi/promin/guias/ProMIN.pdf 
Secretaría de Educación Pública. (2003b). Hacia una politica integral para la formación y el desarrollo profesional de los maestros de educación básica (serie Cuadernos de discusión 1). México: Dirección General de Normatividad / Subsecretaría de Educación Básica y Normal. Recuperado de http://wbgfiles.worldbank.org/ documents/hdn/ed/saber/supporting_doc/LCR/Teachers/Mexico/AS_S6_MEXICO_ BaseDocument-PolicyofTeacherDevelopment_03.pdf

Secretaría de Educación Pública (SEP). (2006). Reglas de operación del Programa de Mejoramiento Institucional de las Escuelas Normales Públicas para el año 2006. Diario Oficial de la Federación. Recuperado de https://www.dgespe.sep.gob.mx/ public/ddi/promin/reglas/reglas 2006.pdf

Secretaría de Educación Pública (SEP). (2012). Acuerdo número 650 por el que se establece el plan de estudios para la formación de maestros de educación preescolar. Diario oficial de la Federación. Recuperado de https://www.sep.gob.mx/work/ models/sep1/Resource/42b00ee7-33da-4bff-85e3-ef45b0f75255/a650.pdf

Secretaría de Educación Pública (SEP). (2018). Acuerdo número 14/07/18 por el que se establecen los planes y programas de estudio de las licenciaturas para la formación de maestros de educación básica que se indican. Diario oficial de la Federación. Recuperado de http://www.apta.com.mx/apta2008/ce/dof/ descargapdf/2018/08Agosto/20180803/sep18080312-1.pdfdiario

Simbaqueba, M.N. (2016). Una aproximación a la nueva gestión pública en Bogotá. Administración \& Desarrollo, 46(2), 200-218. Recuperado de http://esapvirtual.esap. edu.co/ojs/index.php/admindesarro/article/ view/67

Trejo, R.M. y Andrade, R.A. (2013). Evolución y desarrollo de las reformas estructurales en México (1982-2012). El Cotidiano, (177), 37-46. Recuperado de elcotidianoenlinea.com.mx/pdf/17705.pdf

Tonón, G. (2011). La utilización del método comparativo en estudios cualitativos en ciencia política y ciencias sociales: diseño y desarrollo de una tesis doctoral. Kairos, 15(27). Recuperado de https://dialnet.unirioja.es/descarga/articulo/3702607.pdf

Vargas, A.C. (2007). Análisis de las políticas públicas. Perspectivas, (19), 127-136. Recuperado de https://www.redalyc.org/articulo.oa?id=425942453011

Zorrilla, M. (2002). Diez años después del Acuerdo Nacional para la Modernización de la Educación Básica en México: retos, tensiones y perspectivas. Revista Electrónica de Investigación Educativa, 4(2). Recuperado de http://redie.uabc.mx/vol4no2/ contenido-zorrilla.html 
